\title{
Incarcerated Femoral Littre's Hernia After Inguinal Hernia Repair, Report of a Case
}

\author{
Georgios Kampouroglou, ${ }^{\mathrm{a}, \mathrm{b}}$, Baya Huyaga, Slobodan Prica ${ }^{\mathrm{a}}$
}

\begin{abstract}
Meckel's diverticulum is the most common congenital abnormality of the gastrointestinal tract, but still remaining a rare entity $(0.3-$ $3 \%$ ). A protrusion of a Meckel's diverticulum through a potential abdominal opening is called a Littrés hernia. We are reporting a case of an incarcerated right femoral Littré's hernia 6 years after repair of a right inguinal hernia with the Lichtenstein technique.
\end{abstract}

Keywords: Meckel's diverticulum; Littré's hernia; Incarcerated hernia

\section{Introduction}

A Littré's hernia is any hernia containing Meckel's diverticulum, and was described by the French surgeon Alexis Littré in 1700 . He described 3 cases from cadaverous studies of incarcerated femoral hernias containing a diverticulum of the small bowel [1].

Such diverticula are a rare entity occurring in $0.3-3 \%$ of the general population, in males twice as frequently as females, described by Johann Friedrich Meckel in 1812.

It is the most common congenital abnormality of the gastrointestinal tract. It occurs because the remnant of the omphalomesenteric canal, which should be closed by the 7 th, 8th week of gestation, does not close properly. Generally, it remains asymptomatic and it is manifested by its complications (Table 1), the most common of which are gastrointestinal bleeding, inflammation and obstruction [2].

Manuscript accepted for publication July 27, 2012

${ }^{a}$ Department of General Surgery and Traumatology, Jura Hospital,Fbg des Capucins 30, 2800 Delemont, Switzerland

${ }^{\mathrm{b}}$ Corresponding author: Georgios Kampouroglou, Rue de Lyon 16,

1201 Geneva, Switzerland. Email: gkampouroglou@gmail.com

doi: http://dx.doi.org/10.4021/jmc823w

\section{Case Report}

We are reporting a case of a 50-year-old male, who presented in our emergency department with lower abdominal pain with sporadic vomiting episodes during the last three weeks. In the clinical examination we found a right inguinal mass of $5 \mathrm{~cm}$, mobile but irreducible. The intestinal sounds were present but rare and the patient presented no fever and no signs of an acute abdomen. In his surgical history he had a repair with Lichtenstein technique of a right inguinal hernia in 2005 .

In the laboratory tests performed, he presented no leukocytosis neither elevation of CRP. Abdominal CT scan demonstrated a right inguinoscrotal hernia containing small intestine loops and a small quantity of liquid in the hernial sac, a moderate quantity of intraabdominal liquid in the Douglas and dilatation of some intestinal loops (Fig. 1). We proceeded at an emergency surgical exploration, which revealed a right

Table 1. Complications of a Meckel's Diverticulum

\section{Complications of a Meckel's diverticulum}

Haemorrhage

Obstruction

Diverticulitis

Umbilico-enteric fistula

Intussusception

Neoplasia-benign or malignant

Perforation

Peptic ulceration

Littre's hernia 


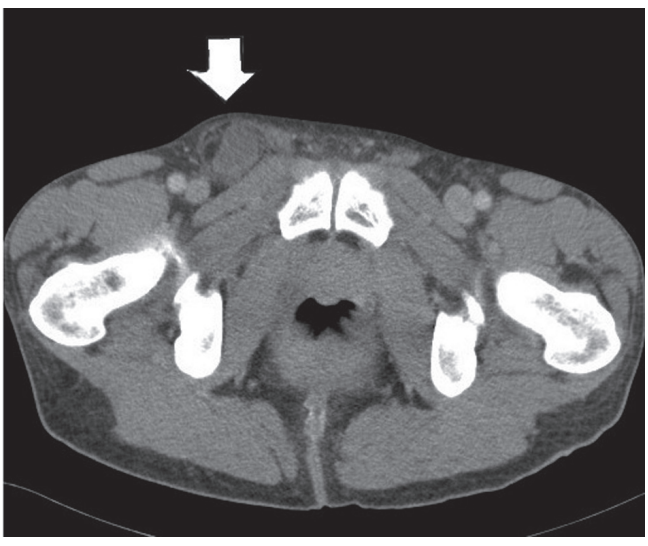

Figure 1. CT scan showing an incarcerated right femoral hernia.

femoral hernia. In the hernial sac we came upon an ileum loop partially incarcerated and a meckel's diverticulum (Fig. 2 ). We continued with a resection of the MD and an ileoileal side to side anastomosis. The femoral hernia was repaired using the Stoppa technique. The patient had an uneventful post operative recovery. He was progressively realimented from the 3 rd postoperative day which he supported very well and was discharged on the 7th postoperative day. He did not present any recurrence in 6 weeks of follow up.

To the best of our knowledge, this is the first reported case in English-language literature of a Littré's femoral hernia after ipsilateral inguinal hernia repair with the Lichtenstein technique.

\section{Discussion}

The incidence of femoral hernia after an inguinal hernia repair with the Lichtenstein technique is rather rare, more frequent in males over sixty years old. Also, the underlying mechanism is supposed to be either an overlooked preexisting femoral hernia or a weakening and enlargement of the femoral canal during inguinal herniorrhaphy [3].

Preoperative diagnosis of an incarcerated Littré's hernia with US or CT is extremely rare and it is normally a peroperative finding [4].

Routine resection of asymptomatic MD is not advised [2]. The gold standard treatment of a Littre hernia is a wedge resection of the MD before the hernia repair [4]. We proceeded to a total resection of the involved loop and to an end to end anastomosis. This surgical approach is preferable when there are signs of intestinal suffering around the MD [2].

Care must be taken not to contaminate the operating field mainly when a mesh is placed for the cure of the hernia. For this reason, in our case, the operating field was carefully cleaned and protected with sterile compresses at the place where the mesh was fixed.

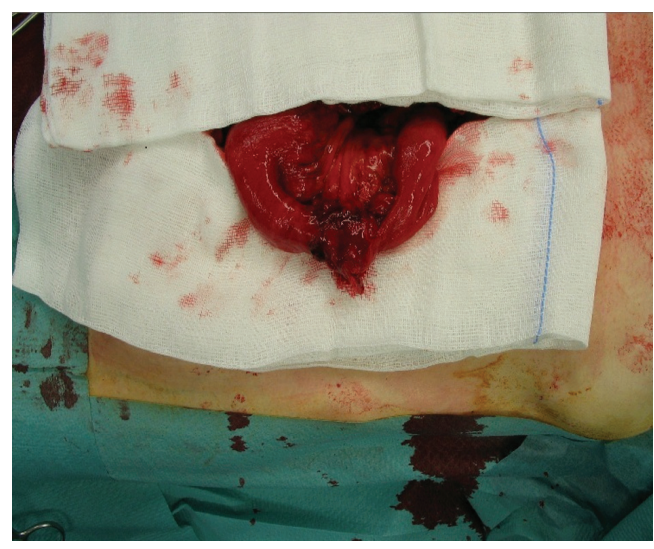

Figure 2. Meckel's diverticulum after opening of the hernia sac.

The use of a mesh is generally not advised when a simultaneous intestinal resection with anastomosis is performed. We proceeded to a pre peritoneal Stoppa repair, after having carefully protected the operating field in the absence of perforation of the loop containing the MD, having also considered this approach as providing better postoperative results concerning recurrence compared to other techniques with no prosthetic mesh use [5].

We conclude that this is a safe procedure for the cure of an incarcerated recurrent femoral Littré's hernia after Lichtenstein repair of an ipsilateral inguinal hernia.

\section{Conflict of Interest}

No conflict of interest.

\section{Grant Support}

No Funding.

\section{References}

1. De Littre M. Observation sur une nouvelle espece de hernie. Histoire de l'Academie Royale des Sciences. 1700;300-310.

2. Skandalakis PN, Zoras O, Skandalakis JE, Mirilas P. Littre hernia: surgical anatomy, embryology, and technique of repair. Am Surg. 2006;72(3):238-243.

3. Mikkelsen T, Bay-Nielsen M, Kehlet H. Risk of femoral hernia after inguinal herniorrhaphy. Br J Surg. 2002;89(4):486-488.

4. Citgez B, Yetkin G, Uludag M, Karakoc S, Akgun I, Ozsahin H. Littre's hernia, an incarcerated ventral incisional hernia containing a strangulated meckel divertic- 
ulum: report of a case. Surg Today. 2011;41(4):576-578.

5. Campanelli G, Pettinari D, Nicolosi FM, Cavalli M,
Avesani EC. Inguinal hernia recurrence: classification and approach. Hernia. 2006;10(2):159-161. 